\title{
Composición del aceite esencial de Tagetes lacera, planta endémica de Baja California Sur, México
}

\section{Composition of essential oil of Tagetes lacera, endemic plant from Baja California Sur, Mexico}

\author{
Francisco Díaz-Cedillo ${ }^{1}$, Miguel A. Serrato-Cruz ${ }^{2 凶}$, Mario Arce-Montoya ${ }^{3}$ y José L. León-de la Luz ${ }^{4}$ \\ ${ }^{1}$ Departamento de Química Orgánica, Escuela Nacional de Ciencias Biológicas, Instituto Politécnico Nacional. Prolongación de Carpio y Plan de \\ Ayala s/n, Casco de Santo Tomás 11340, México, D. F., México. \\ ${ }^{2}$ Departamento de Fitotecnia, Universidad Autónoma Chapingo. Carretera México-Texcoco Km. 37.5, 56230 Chapingo, Estado de México, México. \\ ${ }^{3}$ Laboratorio de Biotecnología, Centro de Investigaciones Biológicas del Noroeste. Apartado postal 128, Mar Bermejo 195 Col. Playa Palo Sta. Rita, \\ 23000 La Paz, Baja California Sur, México. \\ ${ }^{4}$ Herbario, Centro de Investigaciones Biológicas del Noroeste. Apartado postal 128, Mar Bermejo 195 Col. Playa Palo Sta. Rita, 23000 La Paz, Baja \\ California Sur, México. \\ \serratocruz@gmail.com
}

Resumen. Es escasa la información sobre la biología y química de Tagetes lacera Brand. (Asteraceae), especie endémica de Baja California Sur (BCS), México, que por su porte alto y presencia de aroma es una fuente de aceites esenciales útil para el control de plagas y enfermedades de cultivos agrícolas. A partir de partes aéreas de plantas en floración de T. lacera recolectadas en la sierra de la Laguna, BCS se obtuvo aceite esencial mediante hidrodestilación. Por medio del procedimiento de análisis CG-EM, se identificaron 6 compuestos principales: $E$-tagetona (26.2\%), crisantenona (24.8\%), verbenona (22.1\%), $\alpha$-thujeno (20.5\%), $\beta$-pineno (3.1\%) y $\alpha$-pineno (1.9\%).

Palabras clave: Asteraceae, planta endémica, composición del aceite esencial.

\begin{abstract}
It is scarce the background on the biology and chemistry of Tagetes lacera Brand. (Asteraceae), an endemic species from Baja California Sur (BCS), Mexico that considering its plant high and odor is an essential oil source to obtain biopesticides. From aerial parts of plants fully flowered of T. lacera picked in Sierra de la Laguna, BCS, essential oil was obtained by hydrodistillation. Using GC-MS analysis, 6 major compounds were identified: $E$-tagetone (26.2\%), chrysanthenone (24.8\%), verbenone (22.1\%), $\alpha$-thujene (20.5\%), $\beta$-pinene (3.1\%), $\alpha$-pinene (1.9\%).
\end{abstract}

Key words: Asteraceae, endemic plant, essential oil composition.

\section{Introducción}

La distribución de especies del género Tagetes (Asteraceae) en la península de Baja California, México está relacionada con la presencia de climas templados y, a su vez, asociada con la vegetación de pino y pino-encino (Neher, 1965); las especies hasta ahora registradas en esa parte del territorio mexicano corresponden a: T. filifolia Lag., T. lacera Brand., T. lucida L., T. lunulata Ort., T. micrantha Cav. y T. subulata Cerv. (Neher, 1965; Turner, 1996). La sierra de la Laguna, en Baja California Sur es una región en la que se encuentran T. lacera, T. micrantha y $T$. subulata (León-de la Luz et al., 1994); estas especies son aromáticas (Neher, 1965) y el aroma en Tagetes se debe a la presencia de aceites esenciales (López et al., 2008) cuyos metabolitos secundarios no se encuentran identificados

Recibido: 13 julio 2011; 08 noviembre 2011 para la mayoría de las especies mexicanas de ese género. Mientras que T. micrantha y $T$. subulata se distribuyen en otras partes del territorio mexicano (Neher, 1965), T. lacera es endémica de Baja California Sur (Neher, 1965; Turner, 1996); de condición perenne y robusta, de $1.5 \mathrm{~m}$ de altura, crece en altitudes de 2000 a $2200 \mathrm{~m}$ en clima templado en el bosque de encino y pino encino (León-de la Luz et al., 1994; Turner, 1996). Debido al porte alto de la planta y a su naturaleza perenne, la cantidad de biomasa puede ser importante, característica que junto con el aroma intenso y agradable que posee $T$. lacera (Neher, 1965; Turner, 1996; Soule, 1996) hacen promisorio el posible aprovechamiento de los aceites esenciales para uso en aromaterapia o para elaborar biopesticidas, como se ha propuesto para otras especies de Tagetes (Lawless, 1995; Serrato et al., 2007). Desafortunadamente se desconocen aspectos básicos sobre la química de T. lacera, por lo que la identificación de los metabolitos secundarios mayoritarios en el aceite de la especie es imprescindible para contribuir al conocimiento 
de este recurso natural de México y en su eventual aprovechamiento; es así que en el presente trabajo se identificaron algunos componentes del aceite esencial de la parte aérea de plantas de T. lacera.

\section{Materiales y métodos}

En octubre de 2008 se obtuvieron ramas terminales con hojas e inflorescencias de 20 plantas de T. lacera (Fig. $1)$, las cuales se colocaron en recipientes con agua durante $14 \mathrm{~h}$ previo a la destilación. El punto geográfico de la sierra de la Laguna, municipios de La Paz y Los Cabos en Baja California Sur, México donde se obtuvo la muestra, se determinó con geoposicionador Etrex marca Garmin y correspondió a las coordenadas $23^{\circ} 32.76^{\prime} \mathrm{N}$ y $11^{\circ} 00.69^{\prime} \mathrm{O}$, altitud de $1678 \mathrm{~m}$. De acuerdo con información de Conabio (2011), la precipitación anual es de $1800 \mathrm{~mm}$ y clima templado subhúmedo. En el herbario Annetta M. Carter, HCIB, Centro de Investigaciones Biológicas del Noroeste (CIBNOR) están depositados los ejemplares de referencia (sierra de La Laguna, La Cieneguita de La Laguna, BCS: 23.550172N, 109.988163W José Luis León de la Luz 09_196) identificados por el taxónomo José Luis León de la Luz.

Hojas, tallos y cabezuelas de plantas frescas de $T$. lacera se recolectaron in situ y se cortaron en fracciones de $3 \mathrm{~cm}$ para llevarlos a condiciones de hidrodestilación mediante destilador modelo italiano (Serrato-Cruz et al., 2008) durante $45 \mathrm{~min}$; los tejidos se mezclaron y se tuvo un peso de $10 \mathrm{~kg}$ de tejido fresco. No se pesaron los diferentes tejidos.

El aceite se almacenó en un frasco obscuro en condiciones de refrigeración $\left(8{ }^{\circ} \mathrm{C}\right) ; 3$ días después de la extracción se procedió al análisis por cromatografía de gases-espectrometría de masas (CG-EM) en un cromatógrafo de gases marca Polaris Q Finnnigan Trace GC Ultra con un detector de masas Polaris Q (USA), en el modo de impacto electrónico $(70 \mathrm{eV})$. Se empleó una columna RTX-5MX, 5\% difenil-95\% dimetilpolisiloxano de $30 \mathrm{~m}$ $\times 0.25 \mathrm{~mm} \mathrm{D} \times 0.25 \mu \mathrm{m}$. El inyector y el detector de temperatura se establecieron a 250 y $300{ }^{\circ} \mathrm{C}$, respectivamente. La temperatura del horno se inició en $70{ }^{\circ} \mathrm{C}$, se mantuvo 1 min y se programó para alcanzar los $250{ }^{\circ} \mathrm{C}$ con una rapidez de $20^{\circ} \mathrm{C} / \mathrm{min}$. Se utilizó helio como gas acarreador a una velocidad de flujo de $1 \mathrm{ml} / \mathrm{min}$. Se inyectaron muestras diluidas (1/100 en cloruro de metileno, $\mathrm{v} / \mathrm{v})$ de $1 \mu \mathrm{l}$, manualmente en modo split. Los datos cuantitativos se obtuvieron electrónicamente a partir del porcentaje de área de los picos. El intervalo de masas detectadas fue de $35-500 \mathrm{~m} / \mathrm{z}$. Como referencias en el cálculo de los índices de Kovats se emplearon $n$-alcanos. Considerando 3 muestras inyectadas (3 repeticiones o referencias), la identificación de los componentes se realizó comparando los índices de retención relativa, los espectros de masas con la base de datos NIST del sistema GC-MS y los datos espectrales publicados por Allured Publishing Corp., Carol Stream, Illinois, (Adams, 2001).

\section{Resultados}

La cantidad de aceite extraído fue de $25 \mathrm{ml}$ en $10 \mathrm{~kg}$ de tejido fresco $(0.0025 \mathrm{ml} / \mathrm{g})$, de coloración rojiza y de fuerte olor. Los componentes principales en el aceite esencial de $T$. lacera fueron 6 y se ordenaron según su tiempo de elución en la columna capilar (Cuadro 1). De acuerdo con el porcentaje de cada componente en el aceite se tuvo lo siguiente: mayoritariamente $E$-tagetona (26.2\%), crisantenona (24.8\%), verbenona (22.1\%) y $\alpha$-thujeno $(20.5 \%)$, en menor proporción $\beta$-pineno (3.1\%) y $\alpha$-pineno $(1.9 \%)$, y elementos traza (1.4\%) (Fig. 2, Cuadro 1).

\section{Discusión}

Esta es la primera comunicación sobre el aceite esencial de T. lacera, y considerando la cantidad obtenida de $0.0025 \mathrm{ml} / \mathrm{g}$ de plantas in situ, convendrá realizar pruebas piloto de extracción de aceite en destilador de $100 \mathrm{~kg} \mathrm{o}$ más, utilizando plantas en cultivo para evaluar si se conserva el buen rendimiento registrado en plantas in situ. Los compuestos identificados en el aceite de T. lacera corresponden al grupo de monoterpenos acíciclos como $E$-tagetona y los restante son cíclicos (Cropwatch, 2006).

La $E$-tagetona es un componente del aceite esencial en las especies del género, pero en T. terniflora en un porcentaje menor (17\%: Saavedra et al., 2002; 10.7\%: De Feo et al., 2005) o cercano (27\%: Stefanazzi et al., 2006) al registrado en T. lacera (26.2\%; Cuadro 1). La presencia de $E$-tagetona en mayor proporción en el aceite esencial de esta especie es importante para realizar estudios de toxicidad; este compuesto ejerce efecto biológico contra plagas y enfermedades (Saavedra et al., 2002; Stefanazzi et al., 2006) y es un componente del aceite esencial de otras especies de Tagetes (Cropwatch, 2006). El metabolito tagetona pertenece a la serie de los monoterpenos no cíclicos que, junto con otros compuestos como la dehidrotagetona y la ocimenona registrados para Tagetes (Cropwatch, 2006) confieren el olor primario a la mayoría de las especies aromáticas de ese género cuando se frotan o rompen hojas, tallos o cabezuelas (Cropwatch, 2006).

Crisantenona es un compuesto aún no registrado para Tagetes; en el aceite de Artemisia herba-alba constituye el $4.9 \%$ y su actividad antibacteriana se encuentra descrita contra Salmonella, Escherichia y Klebsiella, y antifúngica contra Fusarium y Aspergillus (Zouari et al., 2010). Ver- 
Cuadro 1. Composición mayoritaria de compuestos químicos detectados en el análisis cromatográfico de los aceites esenciales de Tagetes lacera

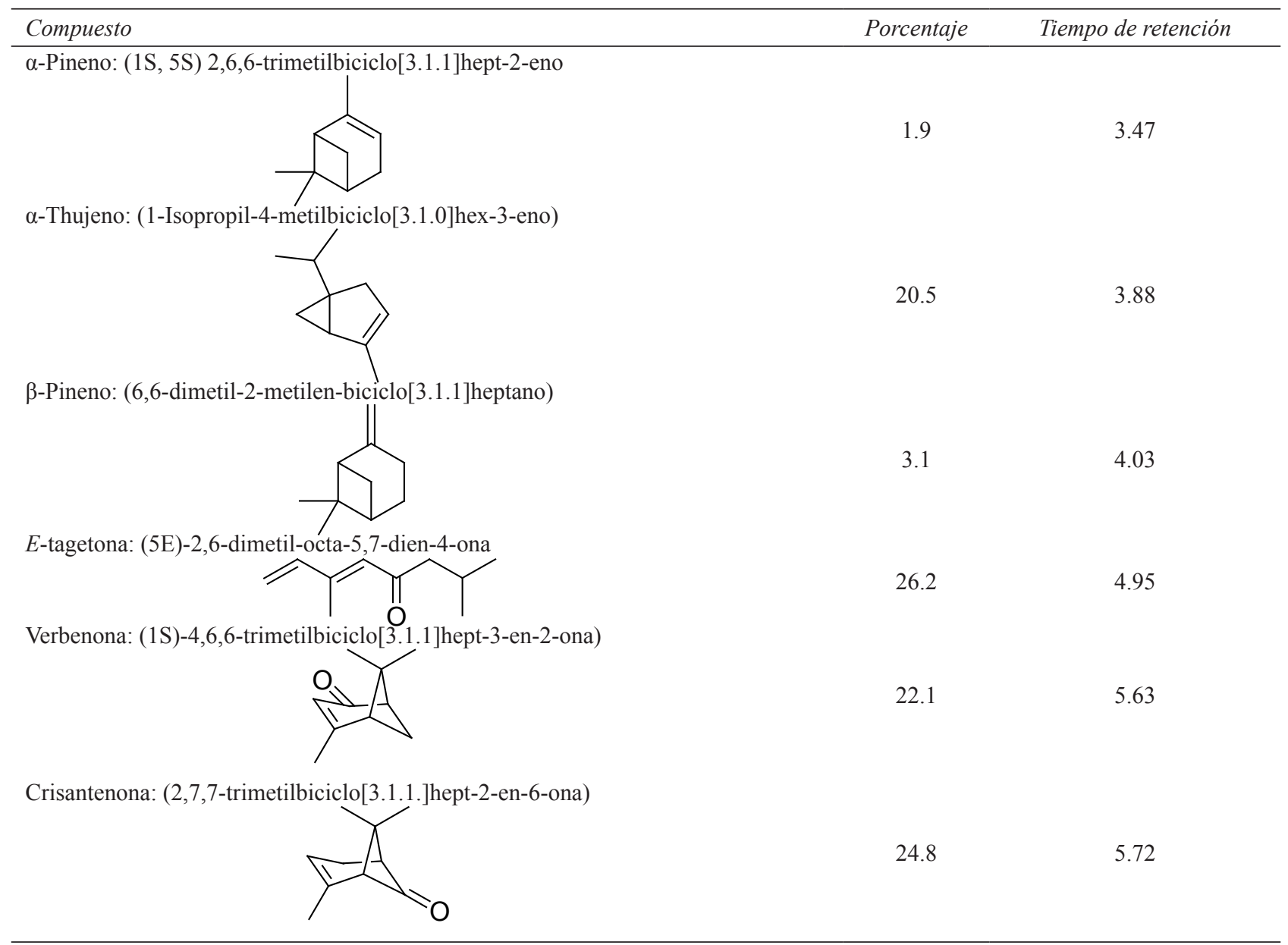

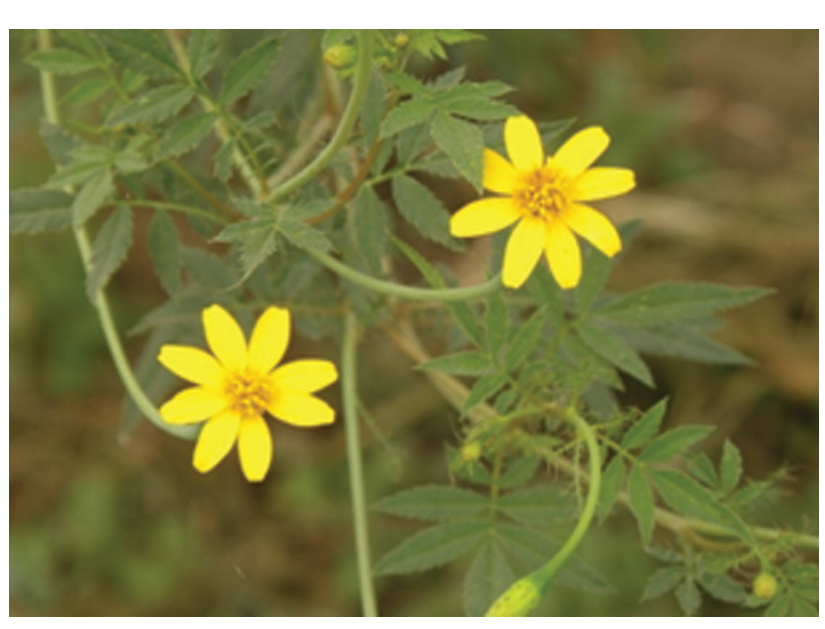

Figura 1. Tagetes lacera Brandegee en floración. (Fotografía: Miguel Ángel Serrato Cruz)

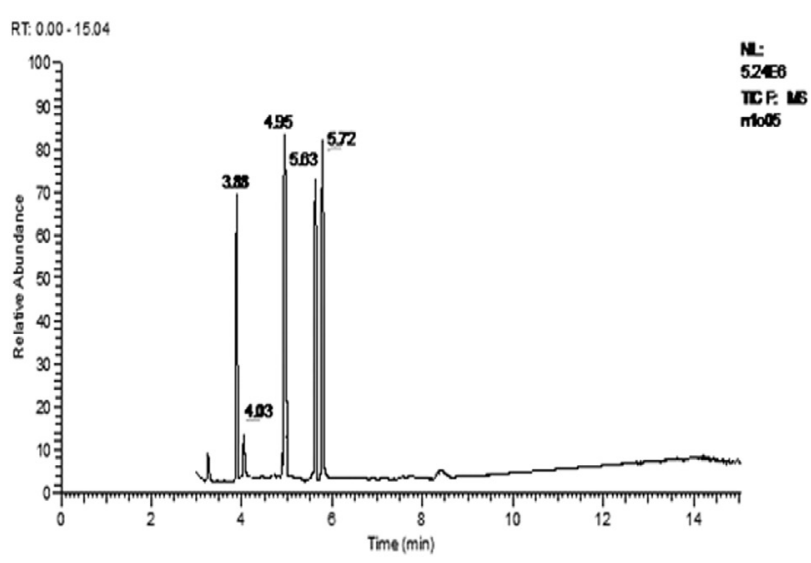

Figura 2. Cromatograma con los picos correspondientes a: $\alpha$-pineno (tiempo de retención, $\mathrm{Rt}=93.4), \alpha$-thujeno $(\mathrm{Rt}=3.8)$, 6,6-dimetil-2-metilenbiciclo[3,1,1]heptano $(\mathrm{Rt}=4.03),(\mathrm{Rt}=4.0)$, E-10 tagetona $(\mathrm{Rt}=4.9)$, verbenona $(\mathrm{Rt}=5.6)$ y crisantenona $(\mathrm{Rt}=5.7)$. 
benona es otro compuesto principal (22\%; Cuadro 1) en el aceite esencial de T. lacera; en T. erecta y T. minuta está en bajo porcentaje (1.5\% a 15\%) (Sefidkon et al., 2004; El-Deeb et al., 2004; Ramaroson-Raonizafinimanana et al., 2009; Meshkatalsadat et al., 2010), por lo tanto, la significativa cantidad de esta sustancia en T. lacera $(22 \%$; Cuadro 1) es prometedora para verificar su posible efecto biológico, pues la presencia del citado compuesto en el aceite de Rosmarinus officinalis L., que alcanza de $27 \%$ a 29\% (Ravid et al., 1997), tiene actividad contra Aspergillus, Bacillus, Candia, Escherichia, Pseudomonas y Staphylococcus (Santoyo et al., 2005) además de constituir una materia prima en tratamientos de aromaterapia (The Ananda Apothecary, 2010). Se conoce que los aceites esenciales que contienen monoterpenos relacionados con la verbenona tienen olores agradables y son utilizados en la elaboración de perfumes (The Ananda Apothecary, 2010), así que T. lacera podría ser una fuente de nuevas fragancias.

El $\beta$-pineno es otro compuesto no registrado en Tagetes que podría ser considerado un quimiotipo o raza química, del cual no se tiene antecedente sobre posibles efectos biológicos, lo cual sugiere estudios sobre su actividad biológica.

Ya se han citado $\alpha$-thujeno y $\alpha$-pineno para Tagetes, que contribuyen a las propiedades antimicrobianas del aceite contra Artemia salina, Pseudomonas aeruginosa y Salmonella thyphi (Senatore et al., 2004; De Feo et al., 2005). En términos generales, el aceite esencial de $T$. lacera lo constituyen monoterpenos cíclicos, destacando el $\alpha$-thujeno, la verbenona y la crisantenona; adicionalmente, la presencia del monoterpeno acíclico tagetona confiere el olor agradable de la mezcla.

Los monoterpenoides contenidos en los aceites esenciales en las plantas tienen funciones de adaptación al medio como protección contra organismos (Wink, 1988) o como importante atrayente de polinizadores (Fabiane et al., 2007); así, los compuestos mayoritarios de T. lacera podrían tener esas funciones en el ambiente donde crece esta especie endémica (Turner, 1996). El conocimiento de los componentes del aceite esencial de T. lacera como planta endémica de Baja California Sur confirma la importancia de conocer la biodiversidad de México, además de contribuir a la discusión sobre el estatus taxonómico de las especies de Tagetes de México (Díaz-Cedillo y SerratoCruz, 2011).

Aunque T. lacera representa un potencial en horticultura ornamental por las cabezuelas grandes y coloridas, y lo durable de la floración (Soule, 1996), el contenido de compuestos en el aceite obtenido de la parte aérea de la planta, permite inferir que la mejor utilidad de esta especie se ubicaría en la industria de la perfumería, la aromate- rapia, la agricultura ecológica y la medicina tradicional, como se consigna en otras especies aromáticas (Santoyo et al., 2005). Se encuentran en progreso estudios sobre la distribución de componentes del aceite esencial según los órganos de la planta de T. lacera y el análisis comparativo entre especies endémicas como T. parryi, T. moorei, T. mulleri, y otras de distribución regional como T. lemmoni para conocer la variación química desde el punto de vista geográfico. Los resultados preliminares del presente estudio sobre el citado recurso natural endémico de Baja California Sur, constituyen una referencia importante para avanzar en otros estudios básicos que, entre otras implicaciones, conduzcan al aprovechamiento de $T$. lacera para proponerla como nuevo cultivo industrial.

\section{Agradecimientos}

La realización de la investigación es una parte del proyecto: "Determinación del rendimiento de aceites esenciales, composición química y características fisicoquímicas de especies aromáticas de Tagetes", el cual corresponde a la Red Tagetes financiada por el Sistema Nacional de Recursos Fitogenéticos (SINAREFI) coordinada por el Sistema Nacional de Inspección y Certificación de Semillas: Programa Sistema Nacional de Recursos Fitogenéticos, Secretaría de Agricultura, Ganadería, Desarrollo Rural, Pesca y Alimentación (SAGARPA).

\section{Literatura citada}

Adams, R. P. 2001. Identification of essential oils components by gas chromatography/quadrupole. Mass spectroscopy. Allured, Carol Stream, Illnois.

Conabio. 2011. Sierra de la Laguna. http://www.conabio.gob.mx/ conocimiento/regionalizacion/doctos/rtp_001.pdf; última consulta: 15.VI.2011.

Cropwatch. 2006. Tagetes oil phototoxicity. Further clarifications. http:/www.cropwatch.org/tageteclarify.htlm; última consulta: 15.VI.2011.

De Feo, V., E. Urrunaga-Soria, R. Urrunaga-Soria y C. Pizza. 2005. Composition and in vitro toxicity of the essential oil of Tagetes terniflora HBK. (Asteraceae). Flavour and Fragrance Journal 20:89-92.

Díaz-Cedillo, F. y M. A. Serrato-Cruz. 2011. Composición del aceite esencial de Tagetes parryi A. Gray. Revista Fitotecnia Mexicana 32:145-148.

El- Deeb, K. S, F. A. Abbas, A. El-Fishawy y J. S. Mossa. 2004. Chemical composition of the essential oil of Tagetes minuta growing in Saudi Arabia. Saudi Pharmacological Journal 12:51-53.

Fabiane, K. C., R. Ferronatto, A. C. dos Santos y S. Becker-Onofre. 2007. Physicochemical characteristics of the essential oils of 
Baccharis dracunculifolia and Baccharis uncinella D. C. (Asteraceae). Revista Brasileira de Farmacognosia 18:197203.

León-de la Luz, J. L., R. Domínguez-Cadena, M. Domínguez-León y R. Coria-Benet. 1994. Flora of the woodlands of the sierra de La Laguna, Baja California Sur, Mexico. In Biodiversity and the management of the Madrean Archipelago: the Sky Islands of Southwestern U. S. and Northwestern Mexico, F. De Lano (ed.). Diane, Darby, Pennsylvania. p. 144-151.

López, M. L., N. E. Bonzani y J. A. Zygadlo. 2008. Allelopathic potential of Tagetes minuta terpenes by a chemical, anatomical and phytotoxic approach. Biochemical and Systematic Ecology 36:882-890.

Lawless, J. 1995. The illustrated encyclopedia of essential oils. Element Books, Rockport, Massachusetts. p. 59, 224.

Meshkatalsadat, M. H., J. Safaei-Ghomi, S. Moharramipour y M. Nasseri. 2010. Chemical characterization of volatile components of Tagetes minuta L. cultivated in south west of Iran by nano scale injection. Digest Journal Nanomatherials Biostruction 5:101-106.

Neher R., T. 1965. Monograph of the genus Tagetes (Compositae). $\mathrm{Ph}$. D. Thesis Indiana University, Bloomington. 306 p.

Ramaroson-Raonizafinimanana, B., P. A. R. Ramanoelina, J. R. E. Rasoarahona y E. M. Gaydou. 2009. Chemical compositions of aerial part of Tagetes minuta L. chemotype essential oils from Madagascar. Journal of Essential Oil Research 21:390392.

Ravid, U., E. Putievsky, I. Katzir, E. Lewinsohn y N. Dudai. 1997. Identification of $(1 R)(+)$-verbenone in essential oils of Rosmarinus officinalis L. Flavour and Fragrance Journal 12:109-112.

Saavedra, N., C. Villa, C. Viturro, A. Molina y S. G. Molina. 2002. Ensayo piloto de extracción de volátiles de Tagetes terniflora H.B.K. http://www.efn.unc.edu.ar/otros/bibliocentro/index_ archivos/31-Tagetes.pdf; última consulta: 12.IX.2010.

Santoyo, S., S. Cavero, L. Jaime, E. Ibanez, F. J. Senorans y G. Reglero. 2005. Chemical composition and antimicrobial activity of Rosmarinus officinalis L. essential oil obtained via supercritical fluid extraction. Journal of Food Protection 68:790-795.

Sefidkon, F., S. Salehyar, M. Mirza y M. Daviri. 2004. The essential oil of Tagetes erecta L. occurring in Iran. Flavour and Perception Symposium 19:579-581.

Senatore, F., F. Napolitano, M. A. Mohamed, P. J. C. Harris, P. N. S. Mnkeni y J. Henderson. 2004. Antibacterial activity of Tagetes minuta L. (Asteraceae) essential oil with different chemical composition. Flavour and Fragrance Journal 19:574-578.

Serrato-Cruz, M. A., J. S. Barajas-Pérez y F. Díaz-Cedillo. 2007. Aceites esenciales del recurso genético Tagetes para el control de insectos, nemátodos, ácaros y hongos. In Agricultura sostenible, vol. 3. Sustancias naturales contra plagas, J. López-Olguín, F. A. Aragón-García, C. RodríguezHernández, y M. Vázquez-García (eds.) Colegio de Postgraduados, Montecillo, Texcoco, Estado de México. p. 142-200.

Serrato-Cruz, M. A., F. Díaz-Cedillo y J. S. Barajas-Pérez. 2008. Composición en el aceite esencial en germoplasma de Tagetes filifolia Lag. de la región centro-sur de México. Agrociencia 42:277-285.

Soule, J. A. 1996. Novel annual and perennial Tagetes. In Progress in new crops, J. Janick (ed.). ASHS Press, Arlington, Virginia. p. 546-551.

Stefanazzi, N., M. M. Gutierrez, T. Stadler, N. A. Bononi y A. A. Ferrari. 2006. Actividad biologica del aceite esencial de Tagetes terniflora Kunth (Asteraceae) en Triboleum castaneum Herbst (Insecta, Coleoptera, Tenebrionidae). Boletín de Sanidad Vegetal de Plagas 32:439-447.

The Ananda Apothecary. 2010. Therapeutic grade essential oils, flower essences and aromatherapy supplies. Rosemary essential oil. http://www.anandaapothecary.com/ aromatherapy-essential-oils/rosemary-verbenone.html; última consulta: 12.VI.2011.

Turner, B. L. 1996. The Comps of Mexico: a systematic account of the family Asteraceae, vol. 6, Tageteae and Anthemideae. Phytologia Memoirs 10:1-93.

Wink, M. 1988. Plant breeding: importance of plant secondary metabolites for protection against pathogens and herbivores. Theoretical and Applied Genetics 75: 225-233.

Zouari, S., N. Zouari, N. Fakhfakh, A. Bougatef, M. A. Ayadi y M. Neffati. 2010. Chemical composition and biological activities of a new essential oil chemotype of Tunisian Artemisia herba-alba Asso. Journal of Medicinal Plants Research 4:871-880. 